02

\title{
Определение функции рассеяния точки компьютерно-синтезированной линзы, сформированной фазовым модулятором света
}

\author{
(C) Н.Г. Сцепуро, Г.К. Красин, М.С. Ковалев ", В.Н. Пестерева \\ Московский государственный технический университет им. Н.Э. Баумана, \\ 105005 Москва, Россия \\ ฯe-mail: m.s.kovalev@gmail.com
}

Поступила в редакцию 21.01.2020 г.

В окончательной редакции 02.03.2020 г.

Принята к публикации 28.03.2020 г.

В различных областях фотоники наблюдается тенденция к переходу от элементов физической оптики к элементам плоской оптики прежде всего из-за уменьшения габаритов приборов и устройств. Наиболее часто для оперативного вывода плоских фазовых элементов используются пространственные модуляторы света. В настоящей работе исследована возможность замены линзовой оптической системы компьютерносинтезированной линзой. Новизна работы заключается в сравнительном анализе функций рассеяния точки линзовой оптической системой и компьютерно-синтезированной линзой. Многочисленные эксперименты подтверждают полученный результат.

Ключевые слова: дифракция, оптическое волновое поле, функция рассеяния точки, компьютерно-синтезированная линза, аберрации, пространственный модулятор света.

DOI: $10.21883 / O S .2020 .07 .49577 .74-20$

\section{Введение}

Одной из важных научно-практических задач современных систем фотоники является необходимость формирования световых полей с заданными пространственными характеристиками [1,2]. С изобретением пространственных модуляторов света (ПМС) появилась возможность для оперативной модуляции амплитуды или фазы светового поля, что имеет особое значение для получения различных световых распределений $[3,4]$. Стоит отметить, что при рассмотрении областей применения различных модуляторов следует исходить из их практических возможностей. Функциональная роль ПМС в современных системах фотоники весьма многогранна: в системах отображения информации [5], в оценке качества световых пучков [6-9], в голографических системах памяти $[10,11]$, в различных корреляторах [12] и т.д.

Несмотря на все вышеперечисленные преимущества, ПМС все еще является относительно новым и малоисследованным оптическим устройством. И пусть за последние годы множество работ было посвящено калибровке модуляторов [13-15], отдельный научный интерес представляет использование не до конца откалиброванных ПМС для вывода типовых оптических элементов, таких как линзы, призмы ит.п. Здесь под не до конца откалиброванными ПМС понимаются ПМС, которые обеспечивают фазовую модуляцию в диапазоне от 0 до $2 \pi$, однако имеют нескомпенсированные искажения, возникающие из-за неравномерностей формы ПМС.

\section{Теория}

При формировании светового поля оптическая система осуществляет в общем случае его пространственноэнергетическое преобразование. Структурное преобразование часто оценивают импульсной реакцией оптической системы. Для исключения масштабного и энергетического преобразования необходимо описывать импульсную реакцию в канонических координатах, а нормированная энергия такой реакции должна быть равна единице. Выполнив вышеозвученные условия, можно говорить про нормированную импульсную реакцию или, иными словами, про функцию рассеяния точки (ФРТ) в задней фокальной плоскости оптической системы

$$
h(x, y)=\left.\frac{1}{H^{\prime}} F^{-1}\left[f\left(\rho_{x}, \rho_{y}\right)\right]\right|^{2},
$$

где $H^{\prime}$ - нормирующая функция, определяемая обобщенной задней светосилой, $f\left(\rho_{x}, \rho_{y}\right)$ - функция зрачка, $F^{-1}$ - оператор обратного преобразования Фурье.

Из выражения (1) можно сделать вывод, что на форму ФРТ влияют функция зрачка (форма апертурной диафрагмы) и неравномерность амплитудного пропускания. Хорошо известно, что профиль распределения интенсивности ФРТ или, другими словами, форма пятна рассеяния определяется только характеристиками оптической системы [16-18]. Например, для линзы радиус пятна рассеяния точки зависит от фокусного расстояния, размера апертуры и длины волны излучения.

Если апертурная диафрагма имеет форму круга, то проходящее через нее излучение будет дифрагировать 


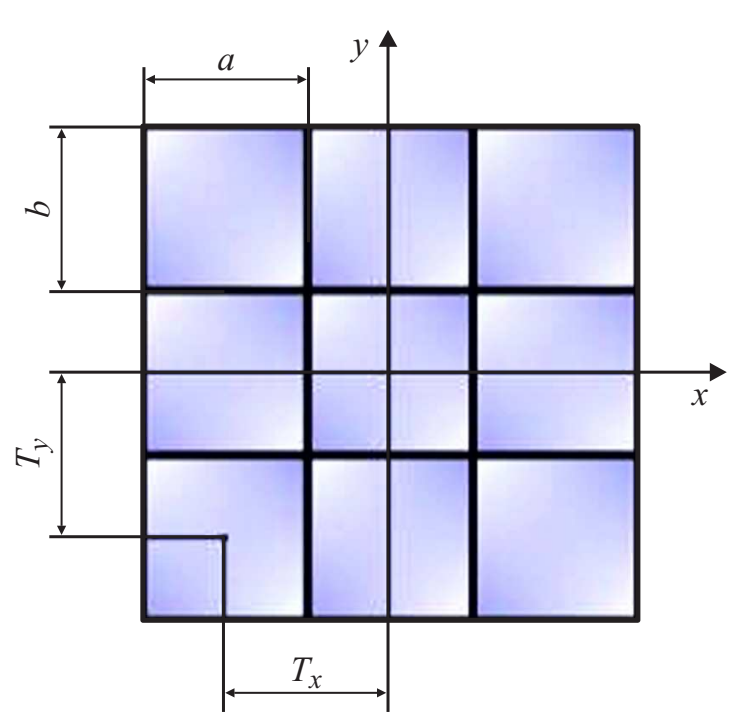

Рис. 1. Структура ПМС.

аналогичным образом, как при дифракции Фраунгофера на круглом отверстии. Дифракционная картина в этом случае будет представлять собой дифракционную картину Эйри с ярким центральным максимумом, известным как диск Эйри. Радиус первого темного кольца картины Эйри можно рассчитать, воспользовавшись уравнением угла $\theta$, на котором возникает первое темное кольцо, заменив $\sin \theta=r / f^{\prime}$ :

$$
r=1.22 \frac{\lambda}{D / f^{\prime}}
$$

где $D / f^{\prime}$ - относительное отверстие, $\lambda$ - длина волны излучения.

Радиус пятна рассеяния точки для выведенной на ПМС компьютерно-синтезированной линзы будет отличаться от физической линзы в силу формы апертурной диафрагмы ПМС и дискретной пиксельной структуры ПМС. Для того чтобы определить именно влияние ПМС на радиус пятна рассеяния точки, необходимо ввести одно ограничение: апертурная диафрагма ПМС имеет форму круга. Это допущение позволит нам исключить влияние формы самого ПМС и акцентировать внимание на его структурных особенностях.

Пусть ПМС освещается пучком с постоянным значением интенсивности. Тогда после отражения от ПМС распределение интенсивности будет иметь вид

$$
I_{\text {out }}(x, y)=I_{0}(x, y) H_{\Pi М С}(x, y),
$$

где $I_{0}(x, y)$ - распределение интенсивности до ПМС, $I_{\text {out }}(x, y)$ - распределение интенсивности после отражения от ПМС, $H_{\Pi \mathrm{MC}}(x, y)$ - функция отражения ПМС.
Функция отражения ПМС в общем виде зависит от геометрических параметров ПМС (рис. 1) и равна

$$
\begin{aligned}
& H_{\Pi \mathrm{\Pi C}}(x, y)=\sum_{k=-\infty}^{+\infty} \sum_{n=-\infty}^{+\infty} \operatorname{rect}\left(\frac{x-k T_{\Pi \mathrm{MC} x}}{a}, \frac{y-n T_{\Pi \mathrm{MC} y}}{b}\right) \\
& =\operatorname{rect}\left(\frac{x}{a}, \frac{y}{b}\right) \otimes \frac{1}{T_{\Pi \mathrm{MC} x}} \frac{1}{T_{\Pi \mathrm{MC} y}} \operatorname{comb}\left(\frac{x}{T_{\Pi \mathrm{MC} x}}, \frac{y}{T_{\Pi \mathrm{MC} y}}\right),
\end{aligned}
$$

где $k$ и $n$ - текущее положение пикселей, $T_{\text {ПмС } x}$ и $T_{\text {Пмсу }}$ - период пикселей ПМС, $a$ и $b$ - размер пикселей ПМС по оси $x$ и $y$ соответственно (рис. 1).

Подставив выражение (4) в (3), получим

$$
\begin{aligned}
& I_{\text {out }}(x, y)=I_{0}(x, y)\left(\operatorname{rect}\left(\frac{x}{a}, \frac{y}{b}\right) \otimes \frac{1}{T_{\Pi \mathrm{MC} x}} \frac{1}{T_{\Pi \mathrm{MC} y}}\right. \\
& \left.\times \operatorname{comb}\left(\frac{x}{T_{\Pi \mathrm{MC} x}}, \frac{y}{T_{\Pi \mathrm{MC} y}}\right)\right) \text {. }
\end{aligned}
$$

Для дальнейшего анализа ФРТ линзы, реализованной на ПМС, необходимо перейти в частотную область, взяв от выражения (5) преобразование Фурье. Распределение интенсивности в частотной области после ряда упрощений и замен будет иметь вид

$$
\begin{aligned}
& \tilde{I}_{\text {out }}\left(v_{x}, v_{y}\right)=\frac{a b}{T_{\Pi \mathrm{MC} x} T_{\Pi \mathrm{MC} y}} \tilde{I}_{0}\left(v_{x}, v_{y}\right)+\frac{a b}{T_{\Pi \mathrm{MC} x} T_{\Pi \mathrm{MC} y}} \\
& \times \sum_{k=-\infty k \neq 0}^{\infty} \sum_{n=-\infty n \neq 0}^{+\infty} \tilde{I}_{0}\left(v_{x}-\frac{k}{T_{\Pi \mathrm{MC} x}}, v_{y}-\frac{n}{T_{\Pi \mathrm{MC} y)}}\right) \\
& \times \operatorname{sinc}\left(\pi a \frac{k}{T_{\Pi \mathrm{MC} x}}, \pi b \frac{n}{T_{\Pi \mathrm{MC} y}}\right) .
\end{aligned}
$$

Из выражения (6) можно сделать вывод, что выходной спектр равен входному спектру, но нормированному на

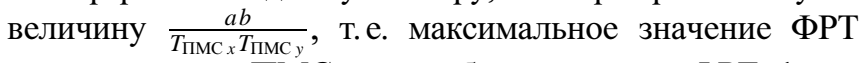
выведенной на ПМС линзы будет меньше ФРТ физической линзы с аналогичными параметрами.

Для регистрации амплитудных распределений широко используются матричные приёмники излучения (МПИ) [19]. Такие приёмники преобразуют непрерывное распределение в дискретное. МПИ не обладают свойствами изопланатичности, так как при поперечном смещении амплитудного распределения относительно МПИ оно не только сместится, но и может изменить свою структуру. Поэтому процесс регистрации амплитудного распределения на МПИ следует рассматривать отдельно.

При составлении полной математической модели МПИ необходимо учитывать его чувствительность, инерционные свойства, постоянную времени приемника, импульсную характеристику, шумовую составляющую ит.д. [20]. Модельно МПИ можно представить в виде пяти блоков: пространственный фильтр, спектральный 


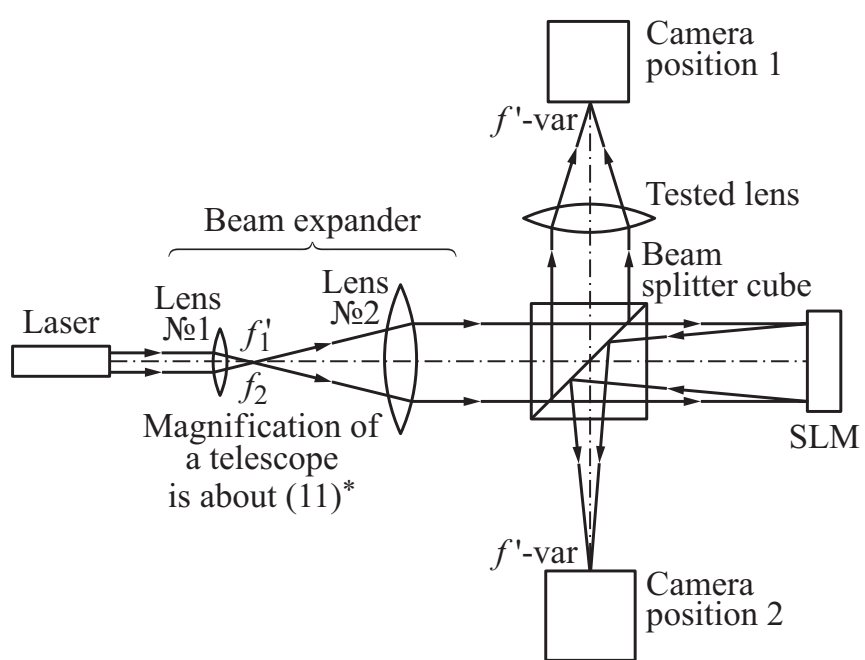

Рис. 2. Схема экспериментальной установки.

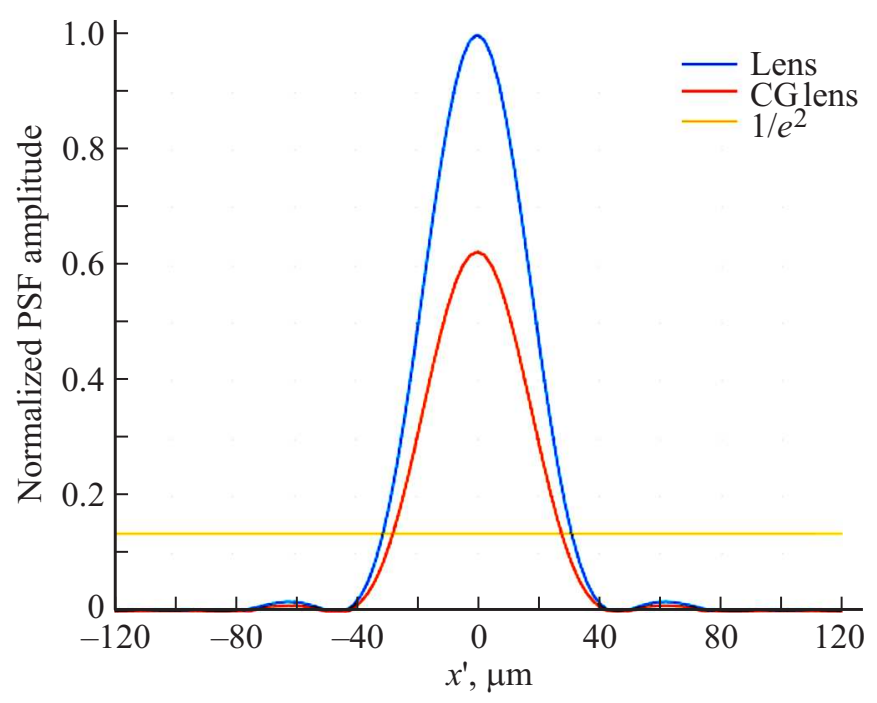

Рис. 3. Теоретическая ФРТ (PSF) для линз с фокусным расстоянием $250 \mathrm{~mm}$.

фильтр, идеальный нешумящий безынерционный приемник, апериодический линейный блок и генератор шума. Однако для нашей задачи составление такой модели МПИ будет избыточно. Поэтому мы остановимся только на первом блоке, а именно на пространственных фильтрующих свойствах МПИ. Пространственные фильтрующие свойства МПИ напрямую зависят от геометрии построения приемника излучения. С геометрической точки зрения МПИ схож с ПМС, схема которого представлена на рис. 1.

Запишем выражение для МПИ в пространственной области в виде

$$
I(x, y)=I_{0}(x, y) \otimes H_{\text {Мпи }}(x, y),
$$

где $I_{0}(x, y)$ - входной сигнал, $I(x, y)$ - выходной сигнал, $H_{\text {мпи }}(x, y)$ - импульсная реакция МПИ.
Значения радиуса пятна рассеяния

\begin{tabular}{c|c|c|c|c}
\hline \multirow{2}{*}{$\begin{array}{c}\text { Фокусное } \\
\text { расстояние }\end{array}$} & \multicolumn{3}{|c}{ Радиус пятна рассеяния, $\mu \mathrm{m}$} \\
\cline { 2 - 5 } mm & \multicolumn{2}{|c|}{ Теория } & \multicolumn{2}{c}{ Эксперимент } \\
\cline { 2 - 5 } & $\begin{array}{c}\text { Оптическая } \\
\text { система }\end{array}$ & КС линза & $\begin{array}{c}\text { Оптическая } \\
\text { система }\end{array}$ & КС линза \\
\hline 250 & 30.9 & 27.6 & 33.7 & 28.1 \\
500 & 61.8 & 55.2 & 67.6 & 56.5 \\
1000 & 123.5 & 110.4 & 127.6 & 113.2
\end{tabular}

Импульсная реакция МПИ, или, другими словами, передаточная функция, имеет вид [21]

$$
\begin{aligned}
H_{\text {МПи }}(x, y)= & {\left[\operatorname{rect}\left(\frac{x}{c}, \frac{y}{d}\right) \otimes \operatorname{sinc}\left(\frac{\pi}{T_{\text {МПИ } x}}, \frac{\pi}{T_{\text {МПИ } y}}\right)\right] } \\
& \times \operatorname{comb}\left(\frac{x}{c}, \frac{y}{d}\right),
\end{aligned}
$$

где $T_{\mathrm{MПи}}$ и $T_{\mathrm{MПи}}-$ период пикселей МПИ, $c$ и $d-$ размер пикселей МПИ по оси $x$ и $y$ соответственно.

\section{Эксперимент}

Для оценки качества компьютерно-синтезированных (КС или CG) линз, сформированных фазовым ПМС, была разработана экспериментальная установка, схема которой представлена на рис. 2. Установка включает в себя полупроводниковый одночастотный лазер Cobolt с рабочей длиной волны $\lambda=659.6 \mathrm{~nm}$, ПМС PLUTO2-VIS-016 $\left(1920 \times 1080\right.$, период $T_{\Pi м \mathrm{C} x}=T_{\Pi \text { Мс } y}=8 \mu \mathrm{m}$, размер пикселя $a=b=7.7 \mu \mathrm{m})$ и монохромную камеру Thorlabs CS2100M $(1920 \times 1080$, размер пикселя $5.04 \mu \mathrm{m})$. Апертура падающего на линзы и ПМС излучения составляет $4.4 \mathrm{~mm}$. В экперименте использовалась оптическая система в виде одиночной линзы (с фокусным расстоянием в 250, 500 и $1000 \mathrm{~mm}$ ), а также с аналогичной КС линзой.

Согласно техническим характеристикам ПМС, который имеет фактор заполнения $0.93\left(a b / T_{\text {ПмС } x} T_{\text {Пмс } y}=\right.$ $=0.93$ ) и коэффициент отражения на рабочей длине волны лазера равный 0.67, коэффициент отражения безразмерная величина ФРТ КС линзы будет в 0.6239 раз меньше по сравнению с ФРТ оптической системы. Если взглянуть на рис. 3, то можно увидеть, что ФРТ на уровне $1 / e^{2}$ для оптической системы шире, чем для КС линзы, но при этом нули функции идентичны. Во втором и третьем столбцах таблицы приведены теоретические значения радиуса пятна рассеяния на уровне $1 / e^{2}$ для всех оптических элементов.

На рис. 4 приведены экспериментальные значения ФРТ для оптических систем и КС линз, а в четвертом и пятом столбцах таблицы приведены значения радиуса пятна рассеяния на уровне $1 / e^{2}$ для оптической системы. 

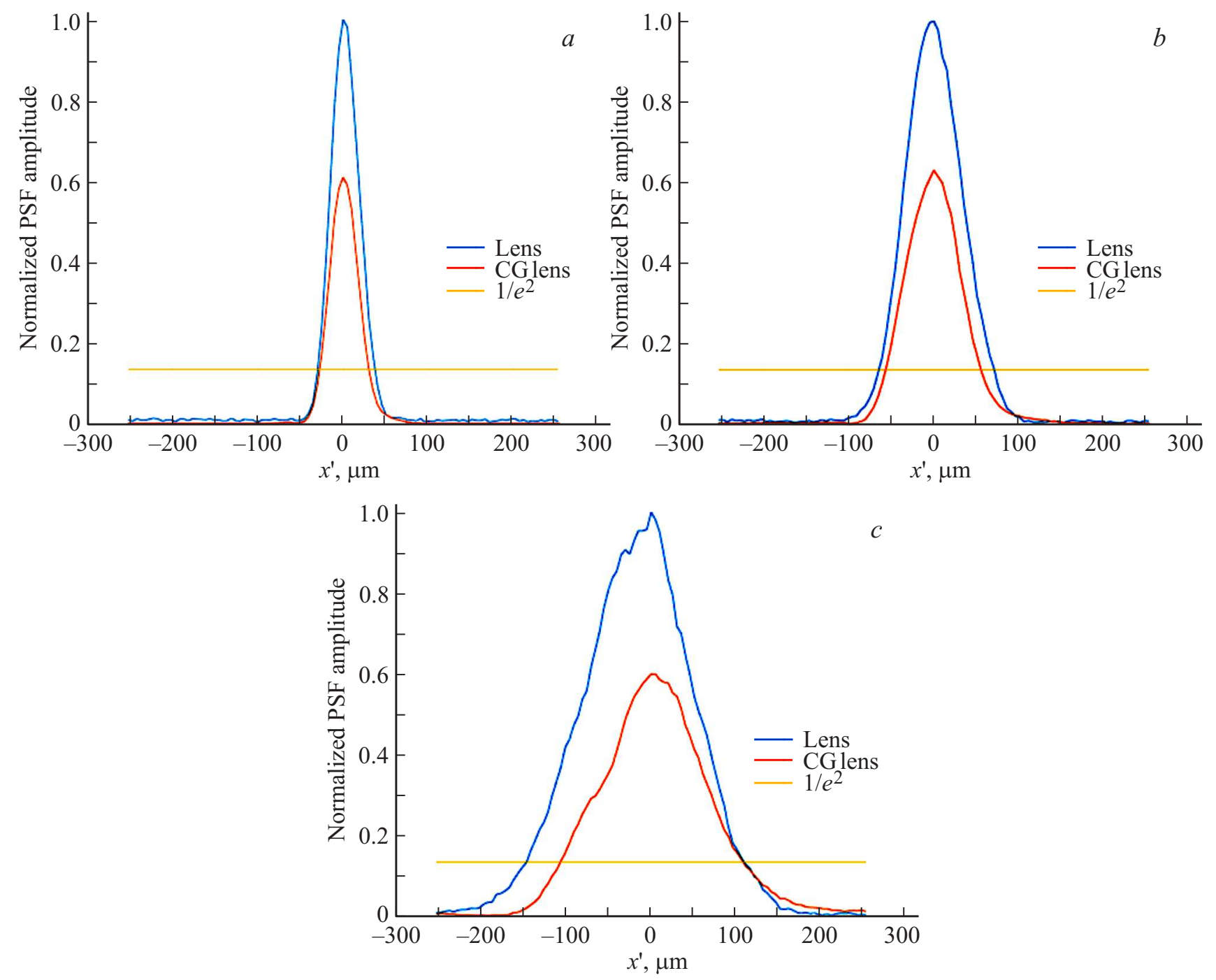

Рис. 4. Экспериментальные значения ФРТ (PSF). $a-$ фокусное расстояние $250 \mathrm{~mm}, b-$ фокусное расстояние $500 \mathrm{~mm}, c-$ фокусное расстояние $1000 \mathrm{~mm}$.

Из таблицы можно сделать вывод, что средний процент отклонения экспериментальных данных от теоретических для оптических систем составил не более $6.7 \%$, а для КС линзы - не более $2.2 \%$.

\section{Выводы}

Представлено теоретическое и экспериментальное исследование качества лазерного излучения, прошедшего через КС линзы, реализованные фазовым ПМС. Проведен сравнительный анализ ФРТ для оптических систем и КС линз. Экспериментально показано, что отклонение от теоретического радиуса пятна рассеяния для КС линз составило порядка $2.2 \%$. Такой результат получен при условии, что ПМС имеет фазовую модуляцию в диапазоне от 0 до $2 \pi$. Свести расхождение к минимуму можно путем компенсации аберраций ПМС [13-15], тем самым исключив влияние неравномерности формы ПМС на выводимые им фазовые распределения. В настоящей работе было показано, что даже не производя компенсацию аберраций ПМС, его можно использовать в качестве альтернативы физической оптической системы.

\section{Конфликт интересов}

Авторы заявляют, что у них нет конфликта интересов.

\section{Список литературы}

[1] Kompanets I.N., Andreev A.L. // Quantum Electron. 2017. V. 47. N 4. P. 294. doi 10.1070/QEL16293

[2] Venediktov V.Y., Nevskaya G.E., Tomilin M.G. // Opt. Spectrosc. 2011. V. 111. N 1. C. 113. doi 10.1134/S0030400X11070216

[3] King S.V., Doblas A., Patwary N., Saavedra G., MartínezCorral M., Preza C. // Appl. Opt. 2015. V. 54. N 29. P. 8587. doi 10.1364/AO.54.008587 
[4] King S.V., Doblas A., Patwary N., Saavedra G., MartínezCorral M., Preza C. // Proc. SPIE. 2014. V. 8949. P. 894913. doi $10.1117 / 12.2040723$

[5] Haist T., Osten W. // J. Micro/Nanolith. MEMS MOEMS. 2015. V. 14. N 4. P. 041310. doi 10.1117/1.JMM.14.4.041310

[6] Kovalev M.S., Krasin G.K., Odinokov S.B., Solomashenko A.B., Zlokazov E.Yu. // Opt. Express. 2019. V. 27. P. 1563. doi 10.1364/OE.27.001563

[7] Žurauskas M., Dobbie I.M., Parton R.M., Phillips M.A., Göhler A., Davis I., Booth M.J. // Optica. 2019. V. 6. P. 370. doi 10.1364/OPTICA.6.000370

[8] Ruchka P.A., Verenikina N.M., Gritsenko I.V., Zlokazov E.Yu., Kovalev M.S., Krasin G.K., Odinokov S.B., Stsepuro N.G. // Opt. Spectrosc. 2019. V. 127. P. 618. doi 10.1134/S0030400X19100230

[9] Васильев А.А., Компанеи, И.Н., Парбенов А.В. // Квант. электрон. 1983. Т. 10. С. 1079-1088.

[10] Zlokazov E.Yu., Odinokov S.B., Verenikina N.M., Donchenko S.S. // Chin. Opt. Lett. 2017. V. 15. N 4. P. 040903. doi 10.3788/COL201715.040903

[11] Zlokazov E.Yu. // Jpn. J. Appl. Phys. 2019. V. 58. P. SKKD04. doi 10.7567/1347-4065/ab2f6c

[12] Goncharov D.S., Zlokazov E.Yu., Petrova E.K., Ponomarev N.M., Starikov R.S. // Bull. Lebedev Phys. Inst. 2019. V. 46. P. 126. doi 10.3103/S1068335619040055

[13] Engström D., Persson M., Bengtsson J., Goksör M. // Opt. Express. 2013. V. 21. N 13. P. 16086.

doi 10.1364/OE.21.016086

[14] Kohler C., Zhang F., Osten W. // Appl. Opt. 2009. V. 48. N 20. P. 4003. doi 10.1364/AO.48.004003

[15] José Luis Martínez Fuentes, Fernández E.J., Prieto P.M., Artal P. // Opt. Express. 2016. V. 24. N 13. P. 14159. doi 10.1364/OE.24.014159

[16] Born M., Wolf E. Principles of Optics. Cambridge University, 1999. 952 р.; Борн М., Воль $\varnothing$ Э. Основы оптики. М.: Наука, 1970. $720 \mathrm{c}$.

[17] Marecnal A., Franson M. Diffraction Structure des Images. Paris, 1960; Марешаль А., Франсон М. Структура оптического изображения. М.: Мир, 1964. 295 с.

[18] Shannon R.R., Wyant J.C. Applied Optics and Optical Engineering. Academic, 1980; Бетенски Э., Хопкинс P., Шеннон Р. и др. / Под ред. Шеннона Р., Вайанта Дж. Проектирование оптических систем. М.: Мир, 1983. 430 с.

[19] Bass M. Handbook of Optics. McGraw-Hill, 1995. 779 p.

[20] Мосягин Г.М., Немтинов В.Б., Лебедев Е.Н. Теория оптико-электронных систем: Учеб. для втузов. М.: Машиностроение, 1990. $431 \mathrm{c}$.

[21] Домненко В.М., Бурсов М.В., Иванова Т.В. Моделирование формирования оптического изображения. СанктПетербург: НИУ ИТМО, 2011. 141 с. 